\title{
1 Detection and phenotypic characterization of carbapenem non susceptible gram-negative bacilli isolated from clinical 3 specimens
}

4 Abera Abdeta ${ }^{1}$, Adane Bitew ${ }^{2}$, Surafel Fentaw ${ }^{1}$, Estifanos Tsige ${ }^{1}$, Dawit Assefa ${ }^{1}$, 5 Tadesse Lejisa ${ }^{3}$, Yordanos Kefyalew ${ }^{4}$ Eyasu Tigabu ${ }^{5}$

${ }^{1}$ National Clinical Bacteriology and Mycology Reference Laboratory, Ethiopian public health institute, Ethiopia.

${ }^{2}$ Department of Medical Laboratory Sciences, College of Health Sciences, Addis Ababa University, Addis Ababa, Ethiopia, ${ }^{3}$ National Clinical Chemistry Reference Laboratory, Ethiopian public health institute, Ethiopia.

${ }^{4}$ Department of Applied Biology, School of Applied Natural Science, Adama Science and Technology University

${ }^{5}$ Global One Health initiative, The Ohio State University, East African Regional Office

Corresponding author:

Email: aberaabdeta4@gmail.com (AB) (1)

5




\section{Abstract}

27 Background: Multi-drug resistant, extremely drug-resistant, pan-drug resistant, carbapenem-

28 resistant, and carbapenemase-producing gram-negative bacteria are becoming more common in health care settings and are posing a growing threat to public health.

Objective: The study was aimed to detect and phenotypically characterize carbapenem non susceptible gram-negative bacilli at Ethiopian Public Health Institute.

Materials and methods: Prospective cross-sectional study was conducted from June 30, 2019, to May 30, 2020, at the national reference laboratory of the Ethiopian Public Health Institute. Clinical samples were collected, inoculated, and incubated in accordance to standard protocol for each sample. Antimicrobial susceptibility testing was done using Kirby Bauer disk diffusion. Identification was done using the traditional biochemical method. Multidrug-resistant and extensively drug-resistant were classified using a standardized definition established by European Centers for Disease prevention and control and the United States Centers for Disease prevention and control experts. Carbapenemase production was confirmed by modified carbapenem inactivation and a simplified carbapenem inactivation method. Meropenem with EDTA was used to differentiate serine carbapenemase and Metallo $\beta$-lactamase.

Results: A total of 1337 clinical specimens were analyzed, of which 429-gram negative bacilli isolates were recovered. Out of 429 isolates 319, 74, and 36 were Enterobacterales, Acinetobacter

44 species, and P. aeruginosa respectively. In our study, the prevalence of Multidrug-resistant, 45 extensively drug-resistant, Carbapenemase-producing, and carbapenem non-susceptible Gramnegative bacilli were, $45.2 \%, 7.7 \%, 5.4 \%$, and $15.4 \%$ respectively. Out of 66 isolates screened for

47 Carbapenemase, 34.8\% (23/66) were Carbapenemase enzyme producers. Ten out of twenty-three 
48 Carbapenemase-positive organisms were Metallo-beta-lactamase producers. Thirteen out of

49 twenty-three isolates were serine carbapenemase producers. Three out of 13 serine Carbapenemase

50 positive organisms were Klebsiella pneumoniae Carbapenemase.

51 Conclusion: The finding from this study revealed a high prevalence of Multidrug-resistant,

52 extremely drug-resistant, carbapenemase-producing gram-negative bacteria, particularly among

53 Intensive care unit patients at the health facility level, this necessitates a robust laboratory-based

54 antimicrobial resistance monitoring and infection prevention and control program.

55

56

57

58

59

60

61

62

63

64

65

66

67 


\section{Introduction}

69 The modern discovery of the antimicrobial agent is considered one of the most fundamental

70 milestones accomplished in the history of medicine and the discovery saved millions of lives [1].

71 Antimicrobials were first used to treat infections in the 1940s [2]. Shortly after the discovery of,

72 Antimicrobials, the fast spread of antimicrobial resistance poses a serious threat to global public

73 health [2].

74 The extensive use of Antimicrobials for treating humans and animal infections along with

75 globalization and international travel leads to the fast spread of resistant strains globally [3].

76 Particularly, increasing incidence of healthcare-associated infections due to multi-drug resistant

77 (MDR), extremely drug-resistant (XDR), carbapenem-resistant, and Carbapenemase-producing

78 Gram-negative bacilli (GNB) has been widely reported [4-7].

79 International subject matter experts came together through a joint initiative by the European Centre for Disease Prevention and Control (ECDC) and the United States Centers for Disease Control and

81 Prevention (CDC), to create a standardized international definition with which to describe acquired

82 resistance profiles [8].

83 They defined MDR as acquired non-susceptibility to at least one agent in three or more

84 antimicrobial categories, XDR as non-susceptibility to at least one agent in all but two or fewer 85 antimicrobial categories (i.e., bacterial isolates remain susceptible to only one or two categories),

86 and PDR as non-susceptibility to all agents in all antimicrobial categories. To apply these

87 definitions, bacterial isolates should be tested against all or nearly all of the antimicrobial agents

88 within the antimicrobial categories and selective reporting and suppression of results should be 89 avoided [8]. 
90 The common mechanism of developing resistance to carbapenem antibiotics is through

91 carbapenemase enzyme production [9]. Carbapenemase is the most versatile family of $\beta$ lactamase

92 and recognizes almost all hydrolyzable $\beta$ lactams, and most are resilient against inhibition by all

93 available $\beta$ lactamase inhibitors [9]. Klebsiella pneumonia Carbapenemase (KPC) hydrolyze

94 penicillin, all cephalosporins, monobactams, carbapenems, and $\beta$ lactamase inhibitors. Metallo- $\beta$ -

95 lactamases usually exhibit resistance to penicillin, cephalosporins, carbapenems, and the clinically

96 available $\beta$ lactamase inhibitors but are inhibited by monobactams [10].

97 There is a considerable knowledge gap regarding risk factors associated with the occurrence of

98 MDR bacteremia [11]. Identifying risk factors for acquiring gram-negative bacteremia could

99 potentially help patient care and management [11].

100 Despite the growing global burden of multidrug resistance, extremely drug-resistant, and 101 carbapenemase-producing gram-negative bacilli, data on multidrug resistance, extremely drug 102 resistance, and carbapenemase-producing organisms that includes both fermentative and non103 fermentative gram-negative bacilli in Ethiopia is scarce. Hence, the objective of this study was to 104 determine the prevalence of MDR, XDR, carbapenem non-susceptible, and carbapenemase105 producing gram-negative bacilli isolated from various clinical specimens, as well as to 106 phenotypically characterize carbapenem non-susceptible isolates, at the Ethiopian Public Health 107 Institute, 


\section{Materials and methods}

\section{Study design, site, and period}

114 A prospective cross-sectional study was conducted from June 30, 2019, to May 30, 2020, at the

115 National Clinical Bacteriology and Mycology Reference Laboratory on clinical samples collected

116 there and referred from different health care settings in Addis Ababa.

\section{Sample collection and processing}

118 Microbiological specimens were collected from the body fluids, ear swabs, sputum, urine, pus, 119 cerebrospinal fluid, blood, and tracheal aspirates and processed following standard procedures. In 120 case sample transportation delay is inevitable appropriate transport media were used. A total of 1211337 clinical specimens were collected during the study period. Specimens collected from each 122 patient were inoculated onto culture media and incubated at appropriate temperatures and periods 123 according to standard protocols related to each sample. Identification was done using the 124 conventional biochemical method. Gram staining, colony characterization, and biochemical tests 125 were done as part of identification. AST was done by Kirby Baur disk diffusion method as per CLSI 126 M100 2020. All frequently isolated Enterobacterales, Acinetobacter species, and P. aeruginosa 127 recovered from the various clinical specimen during the study period were included. All the 128 necessary variables such as socio-demographic (age and sex), type of specimen, referring health 129 facilities, patient location, previous antibiotic exposure from the request form were entered onto 130 pre-configured WHOnet software version 2019. 


\section{Bacterial identification and antimicrobial susceptibility testing}

Bacterial identification: The samples were inoculated onto appropriate culture media,

137 incubated at appropriate temperature and time following standard procedure. The growth was inspected to identify the bacteria. Presumptive identification of bacteria was done based on Gram properties of the organisms for the final identification of the isolates. Gram-negative rods were identified by performing a series of biochemical tests such as carbohydrate fermentation on triple

142 sugar iron agar, Oxidase strips, Simon's citrate agar, and lysine iron agar. Indole production and 143 motility were checked on the sulfide-indole-motility (SIM) medium. Urease production was 144 inspected using a urea agar base supplemented with $40 \%$ urea solution.

Antimicrobial susceptibility testing: Kirby Bauer disk diffusion method was used on Muller Hinton agar to determine antimicrobial susceptibility patterns of the isolates and CLSI M100 2020

147 was used to interpret the results [12].

148 The Antimicrobial discs used for Kirby Bauer disk diffusion method were in the following 149 concentrations: Ampicillin $(10 \mu 1)$, amoxicillin/clavulanic acid(20/10 $\mu 1)$, piperacillin/tazobactam(1 $15000 / 10 \mu 1)$, cefazolin $(30 \mu 1)$, cefuroxime $(30 \mu 1)$, ceftazidime $(30 \mu 1)$, ceftriaxone $(30 \mu 1)$, cefotaxime $(3$

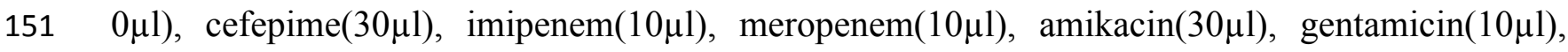
152 tobramycin $(10 \mu 1)$, nalidixic acid $(30 \mu 1)$, ciprofloxacin $(5 \mu 1)$, trimethoprim/sulfamethoxazole

$153(1.25 / 23.75 \mu 1)$, nitrofurantoin $(300 \mu 1)$, and tetracycline $(30 \mu 1)$. 


\section{Detection of Carbapenemase}

155 Clinical and Laboratory Standards Institute CLSI (2010) introduced the modified Hodge test for

156 Carbapenemase detection, but this method can only be used for the accurate detection of KPC-type

157 Carbapenemase in Enterobacteriaceae [13]. CLSI (2012) recommended the Carba NP test method

158 for the detection of Carbapenemase in gram-negative bacilli; however, the preparation of the

159 reagents required for this test is complicated and the solutions cannot be stored for extended periods,

160 limiting its clinical application [14].

161 In 2015 a new detection method, the carbapenem inactivation method (CIM), which is easy to 162 operate and highly sensitive in the detection of Carbapenemase was designed [15]. In 2017, based

163 on the CIM method, CLSI recommended the modified carbapenem inactivation method (mCIM),

164 However; it is a relatively complex method and can only be used to detect Carbapenemase in 165 Enterobacteriaceae and P. aeruginosa [16]. In 2018, based on the mCIM, a simplified carbapenem 166 inactivation method (sCIM) was designed for simple and accurate detection of Carbapenemase in 167 gram-negative bacilli [17]. 


\section{Modified Carbapenem Inactivation method.}

169 In the mCIM, $1 \mathrm{~mL}$ loop full of Enterobacteriaceae or $10 \mathrm{~mL}$ loop full of P. aeruginosa from blood 170 agar plates was emulsified in $2 \mathrm{~mL}$ trypticase soy broth (TSB). A meropenem disk was then

171 immersed in the suspension and incubated for a minimum of $4 \mathrm{~h}$ at $35^{\circ} \mathrm{C}$. A $0.5 \mathrm{McFarland}$ 172 suspension of E. coli ATCC 25922 was prepared in saline using the direct colony suspension method. A Mueller Hinton agar (MHA) plate was inoculated with E. coli ATCC 25922 using the

174 routine disk diffusion procedure. The meropenem disk was removed from the TSB and placed on 175 an MHA plate previously inoculated with the E. coli ATCC 25922 indicator strains. Plates were incubated at $35^{\circ} \mathrm{C}$ in ambient air for $18-24 \mathrm{~h}$. An inhibition zone diameter of 6-15 mm or colonies within a $16-18 \mathrm{~mm}$ zone was considered to be a positive result, and a zone of inhibition of $\geq 19$ $\mathrm{mm}$ was considered to be a negative result [12].

\section{Simplified Carbapenem Inactivation Method}

The sCIM is based on the mCIM with an improvement of experimental procedures. Instead of incubating the antimicrobial disk in the organism culture media for 4 hours as in the mCIM, the organism to be tested was smeared directly onto an antimicrobial disk in the sCIM. To perform the sCIM, for Acinetobacter species, a 0.5 McFarland standard suspension (using direct colony suspension method) of E. coli ATCC 25922 was diluted 1:10 in saline and inoculated onto the MHA plate, following the routine disk diffusion procedure. Plates were allowed to dry for 3-10 $\min [17]$. side of an imipenem disk $(10 \mu \mathrm{g})$; immediately afterward, the side of the disk having bacteria was on an MHA plate was used as the control [17]. 
191 All plates were incubated at $35 \mathrm{C}$ for $16-18 \mathrm{~h}$ in ambient air. Bacterial strains that produced

192 Carbapenemase can hydrolyze imipenem; hence the susceptible indicator strain grew unchecked.

193 Zone of inhibition around the disk shows a diameter of 6-20 mm, or the satellite growth of colonies

194 of E. coli ATCC 25922 around the disk with a zone diameter $\leq 22 \mathrm{~mm}$, was considered

195 carbapenemase positive; a zone of inhibition $\geq 26 \mathrm{~mm}$ was considered to be a negative result; a zone

196 of inhibition of 23-25 mm was considered to be a carbapenemase indeterminate result [17].

\section{Differentiation of Metallo $\beta$-lactamase from serine Carbapenemases}

198 The Modified carbapenem inactivation method positive Enterobacterales (formerly

199 Enterobacteriaceae) and P. aeruginosa and Simplified Carbapenem Inactivation Method positive

200 Acinetobacter species detected were further screened for Class B Metallo-carbapenemase (MBLs),

201 which are characterized by inhibition by metal chelators, EDTA. A $\geq 5$-mm increase in zone

202 diameter for eCIM vs. zone diameter for mCIM was considered a Metallo carbapenemase producer.

$203 \mathrm{~A} \leq 4 \mathrm{~mm}$ increase in zone diameter for the eCIM vs zone diameter of mCIM was considered

204 Metallo-carbapenemase negative. Carbapenemase positive, Metallo carbapenemase negative

205 gram-negative bacilli were considered serine carbapenemase producers [12]

206 Quality control recommendation: K. pneumoniae ATCC® BAA-1705 ${ }^{\mathrm{TM}}$ E. coli ATCC

$20725922^{\mathrm{TM}}$ was used to check the quality of meropenem with EDTA [12]. 
208 Differentiation of Klebsiella pneumoniae carbapenemase (KPC)

209 from other serine Carbapenemases

210 Serine carbapenemase producers were further screened for Klebsiella pneumoniae Carbapenemase

211 (KPC). MIC Test Strip KPC strips consisting of Meropenem (MRP)/Meropenem+Phenylboronic

212 acid (MBO) were used to detect Klebsiella pneumoniae Carbapenemase (KPC) producing gram-

213 negative. [18].

214 Well, isolated colonies from an overnight blood agar plate were suspended into saline to achieve a

$2150.5 \mathrm{McFarland}$ standard turbidity (1 McFarland if mucoid) to obtain a confluent lawn of growth

216 after incubation. The strip was applied to the agar surface with the scale facing upwards and the

217 code of the strip to the outside of the plate. The agar plates were incubated in an inverted position

218 at $35 \pm 2{ }^{\circ} \mathrm{C}$ for $16-20$ hours in the ambient atmosphere. The incubation time was Extend up to 48

219 hours in case of slow-growing Gram-negative non-fermenters [18]

220 Result Interpretation: The ratio of MRP/MBO of $\geq 8$ or $\geq 3 \log 2$ dilutions was interpreted as KPC

221 producer. The phantom zone or deformation of the ellipse was interpreted as positive for KPC

222 regardless of the MRP/MBO ratio [18].

223 Quality control recommendations: E. coli ATCC® 25922 and ATCC® BAA-1705 (intrinsic KPC

224 production) were used to check the quality of KPC strips [18]. 


\section{Data Quality Assurance}

The quality of culture media, antimicrobial disks, and gradient strip were checked as per CLSI, EUCAST guideline, Laboratory SOPs, and manufacturer's instructions.

\section{Data analysis and interpretation}

The data were entered, cleaned, and analyzed using SPSS version 23 and WHOnet 2019 version.

Tables and figures were used to present the results. Chi-square and Univariate analysis were used

to determine the association between multidrug-resistant gram-negative bacilli and different risk factors. P-values less than 0.05 were considered statistically significant.

\section{Ethical considerations}

238 The study was conducted after ethical clearance was obtained from the department research and 239 ethical review committee of the Department of Medical Laboratory Sciences, College of Health 240 Sciences, Addis Ababa University. Official permission from Ethiopian Public Health Institute was

241 obtained. All results were kept confidential; the patient's name and other personal identifiers were encrypted, rather the sample identification number automatically generated by Polytech was used. 


\section{Results}

249 During the study period, 1337 samples were analyzed, of which 429-gram negative isolates were 250 recovered. Of which, 293, 74, and 36 were Enterobacterales, Acinetobacter species, and $P$. 251 aeruginosa were respectively. The number of samples based on specimen types were as follows: 252 Blood (364), Cerebrospinal fluid (46), Ear swabs (28), other body Fluids (30), Pus (366), Sputum 253 (10), Stool (18), Tracheal aspirate (6), and Urine (469). Two hundred thirty-three and one hundred 254 ninety-six-gram negative isolates were recovered from specimens collected from male and female 255 patients respectively.

256 Most of the isolates were isolated from specimens referred from Aabet hospital (187), Ras Desta 257 Hospital (94), and Saint peter Hospital (44). The distribution of gram-negative bacilli among health 258 facilities is summarized in Figure 1.

The highest number of MDR, XDR, and carbapenemase-producing isolates were recovered from specimens referred from Aabet hospital, Ras Desta hospital, and Saint Peter hospital respectively.

262 One hundred eighty-seven (187) gram-negative bacilli were recovered from Aabet hospital 263 specimens, of which $130(69.5 \%), 15(8 \%)$, and 11(5.9\%) were MDR, XDR, and Carbapenemase

264 producers respectively. Ninety-four (94) gram-negative bacilli were recovered from Ras Desta 265 hospital specimens, of which 40(42.5\%),10(10.6\%), and 7(7.4\%) were MDR, XDR, and 266 Carbapenemase producers, respectively. The distribution of MDR, XDR, and carbapenemase267 producing isolates among health facilities was summarized in Figure 2. 
Out of four hundred twenty-nine (429) gram-negative bacilli, clinical isolates analyzed,

$271(\mathrm{~N}=33,7.7 \%)$ were extremely drug-resistant. Out of 33 extremely drug-resistant gram-negative

272 isolates; Acinetobacter species were the predominant isolates $72.73 \%[\mathrm{~N}=24]$. The remaining nine

273 XDR isolates were as follows; K. Pneumonia $9.09 \%[\mathrm{~N}=3]$, P. mirabilis $12.12 \%[\mathrm{~N}=4]$, E. cloacae

$2743.03 \%[\mathrm{~N}=1]$, and $P$. aeruginosa $3.03 \%[\mathrm{~N}=1]$ Table 1 . Out of 33 XDR isolates $[\mathrm{N}=21,63.64 \%]$ were

275 isolated from intensive care units. The highest number of XDR gram-negative bacilli were isolated

276 from urine samples [N=16, 48.48\%]. The distribution of XDR isolates, among specimen types,

277 health facility wards were summarized in Table 1.

Table 1 Distribution of Extremely drug-resistant gram-negative bacilli.

\begin{tabular}{|c|c|c|c|c|c|c|c|c|}
\hline \multicolumn{3}{|c|}{ Distribution of XDR GNB } & \multicolumn{3}{|c|}{$\begin{array}{l}\text { XDR GNB from different } \\
\text { units }\end{array}$} & \multicolumn{3}{|c|}{$\begin{array}{l}\text { XDR GNB based on } \\
\text { specimen sources }\end{array}$} \\
\hline $\begin{array}{l}\text { Organism } \\
\text { name }\end{array}$ & $\mathbf{N}$ & $\%$ & Ward & $\mathbf{N}$ & $\%$ & $\begin{array}{l}\text { Specimen } \\
\text { types }\end{array}$ & $\mathbf{N}$ & $\%$ \\
\hline $\begin{array}{l}\text { Acinetobacter } \\
\text { species }\end{array}$ & 24 & 72.73 & $\begin{array}{l}\text { Intensive } \\
\text { care unit }\end{array}$ & 21 & 63.64 & Urine & 16 & 48.48 \\
\hline K. Pneumonia & 3 & 9.09 & Burn unit & 2 & 6.06 & Blood & 7 & 21.21 \\
\hline P. mirabilis & 4 & 12.12 & Orthopedics & 3 & 9.09 & Sputum & 1 & 3.03 \\
\hline E. cloacae & 1 & 3.03 & Unknown & 6 & 18.18 & CSF & 1 & 3.03 \\
\hline$P$. aeruginosa & 1 & 3.03 & Emergency & 1 & 3.03 & pus & 8 & 24.24 \\
\hline Total & 33 & 100 & Total & 33 & 100 & Total & 33 & 100 \\
\hline
\end{tabular}

281 Out of 429 isolates, 194 were MDR GNB isolates. The most frequently isolated MDR organism 
285 Out of 429 isolates, $15.4 \%$ (66/429) isolates were non-susceptible to either meropenem or

286 Imipenem. Carbapenem non-susceptible isolates were considered a candidate for carbapenemase

287 screening. Out of 66 isolates screened for Carbapenemase, 34.8\% (23/66) were Carbapenemase

288 enzyme producers Table 2. Ten out of twenty-three Carbapenemase-positive organisms were

289 Metallo-beta-lactamase (MBL) producers. Thirteen out of twenty-three isolates were serine

290 carbapenemase producers. Three out of thirteen serine Carbapenemase-positive organisms were

291 Klebsiella pneumoniae Carbapenemase (KPC). Out of 10 Metallo-beta-lactamase positive isolates,

$2925(50 \%)$ were K. Pneumoniae, and the remaining were summarized in Table 2. Three KPC isolates

293 were, E. coli, K. Pneumoniae, and P. aeruginosa Table 2. 
Table 2 Prevalence of multi-drug resistance and Extremely drug resistance, and carbapenemase-producing GNB against 8 antimicrobial classes.

\begin{tabular}{|c|c|c|c|c|c|c|c|c|c|c|c|c|c|c|c|}
\hline Organisms & $\mathrm{R}_{0}$ & $\mathrm{R}_{1}$ & $\mathrm{R}_{2}$ & $\mathrm{R}_{3}$ & $\mathrm{R}_{4}$ & $\mathrm{R}_{5}$ & $\mathrm{R}_{6}$ & $\mathrm{R}_{7}$ & $\mathrm{R}_{8}$ & $\begin{array}{l}\mathrm{N} \% \\
\mathrm{MDR}\end{array}$ & $\begin{array}{l}\text { N\% } \\
\text { XDR }\end{array}$ & $\begin{array}{c}\mathrm{N} \% \\
\text { Carbapenemase }\end{array}$ & $\begin{array}{l}\text { N\% } \\
\text { MBL }\end{array}$ & $\begin{array}{l}\mathrm{N} \% \\
\text { Serine }\end{array}$ & $\begin{array}{l}\mathrm{N} \% \\
\mathrm{KPC}\end{array}$ \\
\hline E. $\operatorname{coli}(N=152)$ & 3 & 52 & 61 & 20 & 14 & 2 & 0 & 0 & 0 & $36(23.7)$ & $0(0)$ & $2(1.3)$ & $1(0.7)$ & $1(0.7)$ & $1(0.7)$ \\
\hline K. pneumoniae $(N=109)$ & 0 & 0 & 36 & 51 & 10 & 9 & 0 & 1 & 2 & $80(73.4)$ & $3(2.8)$ & $9(8.3)$ & $5(4.6)$ & $4(3.7)$ & $1(0.9)$ \\
\hline K. $\operatorname{oxytoca}(N=10)$ & 0 & 0 & 9 & 1 & 0 & 0 & 0 & 0 & 0 & $1(10)$ & $0(0)$ & $0(0)$ & $0(0)$ & $0(0)$ & $0(0)$ \\
\hline K. Ozaenae $(N=11)$ & 0 & 0 & 2 & 4 & 5 & 0 & 0 & 0 & 0 & $2(18.2)$ & $0(0)$ & $2(18.2)$ & $0(0)$ & $2(18.2)$ & $0(0)$ \\
\hline E. $\operatorname{cloacae}(N=11)$ & 0 & 1 & 6 & 1 & 2 & 0 & 0 & 0 & 1 & $4(36.4)$ & $1(9.1)$ & $0(0)$ & $0(0)$ & $0(0)$ & $0(0)$ \\
\hline P. mirabilis $(N=17)$ & 0 & 1 & 10 & 1 & 1 & 0 & 0 & 2 & 2 & $6(35.3)$ & $4(23.5)$ & $1(5.9)$ & $1(5.9)$ & $0(0)$ & $0(0)$ \\
\hline P. vulgaris $(N=3)$ & 0 & 0 & 1 & 1 & 1 & 0 & 0 & 0 & 0 & $2(66.7)$ & $0(0)$ & $0(0)$ & $0(0)$ & $0(0)$ & $0(0)$ \\
\hline M.morganii $(N=6)$ & 0 & 0 & 5 & 0 & 1 & 0 & 0 & 0 & 0 & $1(16.7)$ & $0(0)$ & $0(0)$ & $0(0)$ & $0(0)$ & $0(0)$ \\
\hline $\begin{array}{l}\text { Acinetobacter } \\
\operatorname{species}(N=74)\end{array}$ & 0 & 0 & 22 & 0 & 3 & 5 & 20 & 10 & 14 & $52(70.3)$ & $24(32.4)$ & $5(6.8)$ & $2(2.7)$ & $3(4.1)$ & $0(0)$ \\
\hline P. aeruginosa $(N=36)$ & 0 & 3 & 23 & 5 & 4 & 0 & 0 & 0 & 1 & $10(27.8)$ & $1(2.8)$ & $4(11.1)$ & $1(2.8)$ & $3(8.4)$ & $1(2.8)$ \\
\hline Total $(\mathrm{N}=429)$ & 3 & 57 & 175 & 84 & 41 & 16 & 20 & 13 & 20 & $194(45.2)$ & $33(7.7)$ & $23(5.4)$ & $10(2.3)$ & $13(3)$ & $3(0.7)$ \\
\hline
\end{tabular}

Abbreviations: $\mathrm{R}_{0}-\mathrm{R}_{8}$ : No resistant to antimicrobial class to Resistant to eight antimicrobial classes, MDR-Multi-drug resistant, XDR-

Extensively drug-resistant, MBL-Metallo- $\beta$-lactamase, KPC-Klebsiella pneumoniae carbapenemase, N-Number, \%-percentage. 
Out of 194 MDR GNB isolates, 45\% were isolated from patients admitted to the intensive care unit, $83.4 \%$ were isolated from patients previously exposed to different antimicrobial agents, $28 \%$ were isolated from patients under mechanical ventilation and/or urinary catheterization, and $34.7 \%$ were isolated from patients with hospital-acquired pneumonia and hospital-acquired infection Table 3.

Admission to an intensive care unit (OR:2.75, 95\% CI: 1.92-3.95, P value: 0.001$)$, mechanical ventilation as source of infection and/or urinary catheterization as source of infection (OR:3.6, 95\% CI :2.3-5.6, P value: $<0.001)$, Hospital-acquired infection and/or hospital-acquired pneumonia as a source of infection (OR:2.94, 95\% CI : 1.95-4.44, P value $:<0.001$ ), and recurrent urinary tract infection ( OR:2.75, 95\% CI:1.68-4.46, P value: $<0.001$ ), and sepsis originating from different focus (OR:15.83, 95\% CI :7.66-32.72, P value:0.001) were significantly associated with acquiring multidrug resistant gram-negative bacilli Table 3.

Table 3 Univariate analysis of MDR GNB infection

\begin{tabular}{|l|l|l|l|l|l|l|}
\hline \multirow{2}{*}{ Risk factors } & \multicolumn{2}{l|}{ MDR GNB } & \multicolumn{2}{l|}{ Non-MDR GNB } & & \\
\cline { 2 - 7 } & $(\mathrm{N}=194)$ & $\%$ & $(\mathrm{~N}=235)$ & $\%$ & OR (95 CI $)$ & P-value \\
\hline $\begin{array}{l}\text { Admission to an intensive } \\
\text { care unit(N=193) }\end{array}$ & $(\mathrm{N}=134)$ & 69.4 & $(\mathrm{~N}=59)$ & 30.6 & $2.75(1.92-3.95)$ & 0.001 \\
\hline $\begin{array}{l}\text { Previous Exposure to } \\
\text { Antimicrobials(N=358) }\end{array}$ & $(\mathrm{N}=169)$ & 47.2 & $(\mathrm{~N}=189)$ & 52.8 & $2.79(0.97-1.65)$ & 0.069 \\
\hline $\begin{array}{l}\text { Sepsis of different } \\
\text { focus(N=84) }\end{array}$ & $(\mathrm{N}=75)$ & 89.3 & $(\mathrm{~N}=9)$ & 10.7 & $15.83(7.66-32.72)$ & 0.001 \\
\hline $\begin{array}{l}\text { Mechanical ventilation and } \\
\begin{array}{l}\text { Urinary catheterization } \\
\text { (N=120) }\end{array}\end{array}$ & $(\mathrm{N}=81)$ & 67.5 & $(\mathrm{~N}=39)$ & 32.5 & $3.60(2.3-5.6)$ & $<0.001$ \\
\hline $\begin{array}{l}\text { Recurrent Urinary tract } \\
\text { infection(N=88) }\end{array}$ & $(\mathrm{N}=57)$ & 64.8 & $(\mathrm{~N}=31)$ & 35.2 & $2.75(1.68-4.46)$ & $<0.001$ \\
\hline $\begin{array}{l}\text { Hospital-acquired infection } \\
\text { and hospital-acquired } \\
\text { pneumonia(N=149) }\end{array}$ & $(\mathrm{N}=93)$ & 62.4 & $(\mathrm{~N}=56)$ & 37.6 & $2.94(1.95-4.44)$ & $<0.001$ \\
\hline
\end{tabular}


MDR: Multi-drug resistant, GNB: Gram-negative bacilli, OR: odds ratio, CI: confidence interval, N: Number, \%: percentage.

Out of 23 carbapenemase-positive organisms, 56.5\% (13/23), 26.1\%(6/23), 8.7\% (2/23),8.7\% $(2 / 23)$ were isolated from urine, pus, blood, and tracheal aspirate respectively Table 4 . Out of 23 carbapenemase-positive organisms, $82.6 \%(19 / 23), 17.4 \%(4 / 23)$ were isolated from the patients admitted to the intensive care unit and unknown ward respectively Table 4 . In this, study the prevalence of carbapenem-non susceptible and carbapenemase-producing gram-negative bacilli is $15.4 \%(66 / 429)$ and 5.4\% (23/429) respectively Table 4.

Table 4 Distribution of carbapenemase among wards and specimen sources

\begin{tabular}{|c|c|c|c|c|c|c|c|}
\hline \multirow{2}{*}{$\begin{array}{l}\text { Specimen } \\
\text { types }\end{array}$} & \multicolumn{3}{|c|}{ Carbapenemase } & \multirow{2}{*}{ Wards } & \multicolumn{3}{|c|}{ Carbapenemase } \\
\hline & Positive & Negative & Total & & Positive & Negative & total \\
\hline Blood & 2 & 7 & 9 & Emergency & 0 & 6 & 6 \\
\hline Pleural fluid & 0 & 1 & 1 & $\mathrm{ICU}$ & 19 & 22 & 41 \\
\hline Pus & 6 & 11 & 17 & Inpatient & 0 & 3 & 3 \\
\hline $\mathrm{CSF}$ & 0 & 2 & 2 & outpatient & 0 & 2 & 2 \\
\hline $\begin{array}{l}\text { Tracheal } \\
\text { aspirate }\end{array}$ & 2 & 3 & 5 & Unspecified & 4 & 10 & 14 \\
\hline tissue & 0 & 1 & 1 & Total & 23 & 43 & 66 \\
\hline urine & 13 & 18 & 31 & & & & \\
\hline total & 23 & 43 & 66 & & & & \\
\hline & ICL & Intensive care & nit, $\mathrm{CSF}$ & Cerebrospina & luid & & \\
\hline
\end{tabular}




\section{Antimicrobial resistance profile of Enterobacteriaceae}

Out of 429 gram-negative isolates, 293 were Enterobacteriaceae family (E. coli, K. Pneumoniae, K. oxytoca, K. ozaenae) Table 2. The highest resistance was observed against Ampicillin by E. coli [89.3\%] Table 5. K. Pneumoniae, K. oxytoca, and K. ozaenae were intrinsically resistant to ampicillin hence not tested against them [12].

K. ozaenae showed $100 \%$ resistance to first, second, and third generation cephalosporins, as well as Trimethoprim-sulfamethoxazole, and E. cloacae showed $100 \%$ resistance to ceftriaxone.The overall resistance profile of Enterobacteriaceae to extended-spectrum cephalosporins ranges from Ceftriaxone [67.7\%], Cefotaxime [73.6\%], ceftazidime [73.6\%], and Cefepime [66.5\%]. E. coli showed high resistance to extended-spectrum cephalosporins; ceftriaxone [63.8\%], cefotaxime [68.6\%], Ceftazidime [65\%] and cefepime [55.7\%] Table 5. K. Pneumoniae showed high resistance to extended-spectrum cephalosporins; ceftriaxone [88.7\%], cefotaxime [82.8\%], ceftazidime [81.1\%], cefepime [81.8\%].

E. coli showed high resistance to ciprofloxacin [62.9\%] and Trimethoprim-sulfamethoxazole [76.7\%], likewise $K$. Pneumoniae showed high resistance to ciprofloxacin [62.3\%] and Trimethoprim-sulfamethoxazole [82.7\%] Table 5. The overall prevalence of Enterobacteriaceae resistance to gentamycin, tobramycin, amikacin is $41.9 \%, 37.5 \%$, and $6.1 \%$ respectively. The overall prevalence of meropenem and imipenem resistance among the Enterobacteriaceae family was $4.2 \%$ and $6.5 \%$ respectively. 


\section{Antimicrobial resistance profile of Morganellacea family}

M.morganii, P. mirabilis, and P. vulgaris were reorganized to Morganellacea families [13]. A total of 26 Morganellacea families were isolated during the study period Table 2. M.morganii and $P$. vulgaris were intrinsically resistant to ampicillin, cefazolin, and cefuroxime and were not reported in Table 5. M.morganii, P.mirabilis, and P.vulgaris intrinsically to Antimicrobials were summarized in Table 5 as Not applicable [NA].

P. mirabilis showed $100 \%$ resistance to Ampicillin, Nalidixic acid, and Trimethoprimsulfamethoxazole, likewise M. morganii showed $100 \%$ resistance to Nalidixic acid and Trimethoprim-sulfamethoxazole. family showed high resistance percentage to ceftriaxone [80.8\%], cefotaxime [61\%], ceftazidime [57.7\%], and cefepime [63.6\%]. The percentages of Meropenem and Imipenem resistance were $14 \%$ and $14.3 \%$ respectively Table 5. The Antimicrobial susceptibility patterns of the Morganellacea family were summarized in Table 5. The percentage of Morganellacea family resistance among aminoglycosides ranges from $26.3 \%$ to $47.5 \%$ Table 5 .

\section{Antimicrobial resistance profile of Acinetobacter species}

Acinetobacter species shows the high percentage of resistance to cefotaxime [95.7\%], ceftriaxone [94.7], ceftazidime [93\%], tetracycline [92.9\%], and cefepime [86.1\%]. Acinetobacter species showed lowest percentage of resistance to Amikacin [29.4\%], Meropenem [48.1\%], Imipenem [29.4\%] Table 5.

\section{Antimicrobial susceptibility patterns of $P$. aeruginosa}

A total of $36 P$. aeruginosa isolates were isolated during the study period. The highest percentage of resistance by $P$. aeruginosa was observed against Ciprofloxacin $[\mathrm{N}=32,21.9 \%$ ], Cefepime $[\mathrm{N}=32,21.7 \%]$, and Meropenem [N=31, 19.4\%] Table 5. 
Table 5 Antibiotic susceptibility pattern of gram-negative bacilli against different antimicrobial classes

\begin{tabular}{|c|c|c|c|c|c|c|c|c|c|c|c|}
\hline & & E. coli & $\begin{array}{l}\text { K. } \\
\text { pneumoniae }\end{array}$ & $\begin{array}{l}\text { K. } \\
\text { oxytoca }\end{array}$ & $\begin{array}{l}\text { K. } \\
\text { ozaenae }\end{array}$ & $\begin{array}{l}\text { E. } \\
\text { cloacae }\end{array}$ & $\begin{array}{l}P . \\
\text { mirabilis }\end{array}$ & P. vulgaris & M.morganii & $\begin{array}{l}\text { Acinetobacter } \\
\text { species }\end{array}$ & P. aeruginosa \\
\hline \multirow{3}{*}{$\begin{array}{c}\text { Ampicillin \& } \beta- \\
\text { Lactam } \\
\text { combinations }\end{array}$} & AMP \%R & 89.3 & $\mathrm{NA}^{*}$ & NA* & $\mathrm{NA}^{*}$ & NA* & 100 & NA* & NA* & NA* & NA* \\
\hline & $\mathrm{AMC} \% \mathrm{R}$ & 33.8 & 64.4 & 33.3 & 87.5 & NA* & 38 & 0 & NA* & NA* & NA* \\
\hline & $\mathrm{TPZ} \% \mathrm{R}$ & 13.3 & 33.3 & 14.3 & 66.7 & 42.9 & 30 & 0 & 17 & 85.4 & 12.5 \\
\hline \multirow{2}{*}{$\begin{array}{c}\text { 1st and 2nd } \\
\text { generation } \\
\text { cephalosporins }\end{array}$} & $\mathrm{CZ} \% \mathrm{R}$ & 60.4 & 85.7 & 57.1 & 100 & NA* & 80 & $\mathrm{NA}^{*}$ & NA* & NA* & NA* \\
\hline & CXM \%R & 64.1 & 91.4 & 60 & 100 & 66.7 & 71 & NA* & $\mathrm{NA}^{*}$ & NA* & NA* \\
\hline \multirow{4}{*}{$\begin{array}{c}\text { Extended- } \\
\text { spectrum } \\
\text { cephalosporins }\end{array}$} & CRO \%R & 63.8 & 88.7 & 50 & 100 & 100 & 69 & 0 & 67 & 94.7 & NA* \\
\hline & $\mathrm{CTX} \% \mathrm{R}$ & 68.6 & 82.8 & 60 & 100 & 25 & 68 & 0 & 67 & 95.7 & $\mathrm{NA}^{*}$ \\
\hline & $\mathrm{CAZ} \% \mathrm{R}$ & 65 & 81.1 & 33.3 & 100 & 71.4 & 83 & 0 & 0 & 93 & 14.3 \\
\hline & FEP $\% \mathrm{R}$ & 55.7 & 81.8 & 40 & 85.7 & 75 & 89 & 0 & 0 & 86.1 & 21.7 \\
\hline \multirow[b]{2}{*}{ Carbapenems } & IPM \%R & 0 & 6.2 & 0 & 50 & 50 & 20 & 0 & 0 & 29.4 & 0 \\
\hline & MEM \%R & 2.2 & 5.2 & 0 & 20 & 9.1 & 14 & 0 & 25 & 48.1 & 19.4 \\
\hline $\begin{array}{l}\text { Folate Pathway } \\
\text { antagonists }\end{array}$ & SXT \%R & 76.7 & 82.7 & 66.7 & 100 & 81.8 & 88 & 66.7 & 100 & 68.4 & NA* \\
\hline \multirow{2}{*}{$\begin{array}{c}\text { Quinolone and } \\
\text { Fluoroquinolone }\end{array}$} & $\mathrm{NA} \% \mathrm{R}$ & 83.3 & 80 & $\mathrm{NA}^{*}$ & $\mathrm{NA}^{*}$ & 0 & 100 & 0 & 100 & NA* & NA* \\
\hline & $\mathrm{CIP} \% \mathrm{R}$ & 62.9 & 62.3 & 25 & 87.5 & 66.7 & 71 & 50 & 60 & 77.6 & 21.9 \\
\hline Tetracycline & TET $\% \mathrm{R}$ & 84.8 & 60 & 66.7 & 60 & 50 & $\mathrm{NA}^{*}$ & NA* & 0 & 92.9 & 0 \\
\hline Nitrofuran & $\mathrm{FM} \% \mathrm{R}$ & 3.1 & 46.4 & 33.3 & 75 & 0 & $\mathrm{NA}^{*}$ & NA* & $\mathrm{NA}^{*}$ & $\mathrm{NA}^{*}$ & NA* \\
\hline \multirow{3}{*}{$\begin{array}{c}\text { Aminoglycoside } \\
\mathrm{s}\end{array}$} & GEN \%R & 20.5 & 71.2 & 0 & 66.7 & 60 & 25 & 0 & 33 & 72.7 & 0 \\
\hline & TOB \% $\mathrm{R}$ & 33.6 & 37.2 & 25 & 72.7 & 57.1 & 63 & 0 & 25 & 54.3 & 17.2 \\
\hline & AMK \%R & 3.1 & 11.1 & 0 & 12.5 & 0 & 46 & 0 & 0 & 29.5 & 4.3 \\
\hline
\end{tabular}

Abbreviations: AMP-Ampicillin, AMC-Amoxicillin-clavulanate, TZP-Piperacillin-tazobactam, CZ-Cefazolin, CXM-Cefuroxime, CRO-Ceftriaxone, CTX-Cefotaxime, CAZ-Ceftazidime, FEP-Cefepime, IPM-Imipenem, MEM-Meropenem, SXT-Trimethoprimsulfamethoxazole, NA- Nalidixic acid, CIP-Ciprofloxacin, TET-Tetracycline, FM-Nitrofurantoin, GEN-Gentamycin, TOBTobramycin, AMK-Amikacin, \%R-Percent resistant, NA*-Not Applicable,0-Zero resistance. 


\section{Discussion}

The prevalence of XDR, MDR, Carbapenemase-producing, and Carbapenem-resistant GNB is increasing $[5,6,19]$. In our study, the prevalence of MDR, XDR, Carbapenemase-producing, and carbapenem non-susceptible GNB is high. The most frequently isolated XDR organisms were Acinetobacter species 32.4\% [24/74] which disagree with the study findings of Beyene et al (E. coli 18.1\% was the dominant XDR GNB followed by K. pneumoniae 11.1\% [19] and Gashaw et al (Klebsiella species 43.3\% was the dominant XDR GNB) [20], the difference could be attributed to geographical differences, the number of samples studied, or the types of gram-negative bacteria considered. In the present study, the highest number of XDR organisms were recovered from urine samples $48.48 \%$ [16/33] and patients admitted to an intensive care unit $63.64 \%$ [21/33].

In the present study, the prevalence of XDR gram-negative bacilli was 7.7\% [33/429], which is slightly lower than study at Ethiopian Public Health Institute, Ethiopia by Beyene et al 8.8\% [19] the variation might be due to the fact the investigators analyzed only Enterobacterales, much lower than the findings from the study at Jimma, Ethiopia by Gashaw et al 41.3\% [20], Arsho Advanced Medical Laboratory, Addis Ababa, Ethiopia by Bitew et al 34.3\% [21], Tertiary Care Hospital, Pakistan by Abbas et al 64\% [22]. The variation might be due to geographic location, the technique utilized, XDR definition, types of organism, etc.

In our study, the prevalence of Carbapenemase-producing gram-negative bacilli was 5.4\%, which is higher than the prevalence of study conducted at the University of Gondar, Ethiopia by Eshetie et al 2.72\% [23] and Ethiopian Public health Institute, Ethiopia by Beyene et al 2\% [19]. However, our result was lower than the result of the study conducted at Tikur Anbessa specialized hospital, Ethiopia by Melese et al 12.12\% [24], Three Hospitals in Amhara region, Ethiopia by Moges et al 
15.7\% [25], Felegehiwot Hospital, Ethiopia by Moges et al 16.2\% [26], Sidama, Ethiopia by Alemayehu et al 9\% [27], Mulago National Referral Hospital, Uganda by Okoche et al 22.4\% [28], and data from laboratories in Spain by Lopez-Hernandez et al 62\% [29]. The variation might be due to the technique utilized, modified carbapenem inactivation method was utilized in our investigation unlike other investigators who used the modified Hodge test, the number of bacterial isolates analyzed, geographic location. However, our study findings are in line with Prospective Cross-Sectional Study conducted at Felegehiwot Hospital, Ethiopia by Alebel et al 5.2\% [30]. The dominant carbapenemase-producing gram-negative bacilli were Klebsiella Pneumoniae 8.3\% [9/109] followed by Acinetobacter species 6.8\% [5/74], the study results marginally coincides with the findings of the following researchers which demonstrated the dominant prevalence of carbapenemase-producing Klebsiella pneumoniae: Beyene et al Klebsiella pneumoniae 5.6\% [19], Melese et al Klebsiella pneumoniae 10.5\%[24], Moges et al Klebsiella pneumoniae 5.8\%[25], Moges et al Klebsiella pneumoniae 10.1\%[26], Lopez-Hernandez Klebsiella pneumoniae 45\% [29]. The present study disagrees with finding by Okoche et al, which showed E. coli as the highest carbapenemase-producing organisms [28], The discrepancy could be explained by the fact that they utilized Boronic acid-based inhibition, a modified Hodge and EDTA double combination disk test, the number of samples analyzed, and a different geographical location. , etc.

The highest number of carbapenemase producing isolates were recovered from urine samples $13 / 23(56.5 \%)$, which strongly disagrees with the results of study conducted by Moges et al in which the highest number of carbapenemase producing isolates was recovered from blood samples $22 / 24(91.6 \%)[25]$

The prevalence of MDR was $45.2 \%$ which is much lower than the study conducted at Ethiopian Public Health Institute, Ethiopia by Beyene et al 94.5\% [19], three referral hospitals, Ethiopia by 
Moges et al 85.8\% [25], Felegehiwot Hospital, Ethiopia by Moges et al $80 \%$ and by Alebel et al 81.1\% [26,30], Addis Ababa, Ethiopia by Teklu et al 68.3\% [31], North Iran by Hemmati 62.8\% [32], and Jimma medical center, Ethiopia by Biset et al 56.67\% [33]. The variation might be due to the study population, the number of isolates assessed, the technique utilized, etc. However, our study findings are in line with the study conducted at Arsho Advanced Medical Laboratory, Addis Ababa, Ethiopia by Bitew et al 42.1\% [21]. The dominant MDR isolates were K. Pneumoniae 80(73.4\%) however our result disagrees with study findings of Beyene et al in which E. coli (99.3\%)is the dominant one followed by K.pneumoniae (90.3\%) [19] the observed variation could be attributable to the study period and proportion of bacterial isolates, Alebel et al in which Acinetobacter species(100\%), P. aeruginosa(100\%), Citrobacter species(100\%), and Enterobacter cloacae(100\%) were the dominant MDR isolates[30], the variation could be attributable to the fact that there were fewer samples evaluated, that samples were taken from intensive care unit patients, or that the study was conducted in a different geographic location and Biset et al [33] the difference might be since they only analyzed urine samples among pregnant women. Likewise, the following researchers reported the dominant prevalence of MDR Klebsiella species Moges et al Klebsiella species (30.6\%) [25], Moges et al. K. pneumoniae (53.3\%) [26] and, Teklu et al K. pneumoniae $(83.5 \%)[31]$

The prevalence of carbapenem carbapenem-resistant gram-negative bacilli is, $10.7 \%(46 / 429)$, in which Acinetobacter species account for about 39 percent of carbapenem-resistant isolates, the finding contradicts the study findings of the following researchers: Beyene et al 1.7\% [19], Alebel et al 21\% [30], Teklu et al 5.2\% [31], Gashaw et al 25\% [20], Melese et al 12.2\% [24], in $K$. pneumoniae accounts for $100 \%, 40 \%, 50 \%, 36 \%$, and $75 \%$ of the gram-negative bacilli isolates respectively, the observed difference could be attributed to the types of gram-negative bacteria 
analyzed, as most of them only included Enterobacteriaceae, the techniques used, geographical location, and so on.

The total resistance profile of Enterobacterales to extended-spectrum cephalosporins ranges from $57.7 \%$ to $80.8 \%$, which marginally agrees with results of study conducted by Beyene et al. $73.5 \%$ to $73.9 \%$ [19], but higher than the study findings of Teklu et al (60.3\% to $62.2 \%)$ [31] and Moges et al $(60.3 \%$ to $66 \%$, in 2021$)$ [25] lower than the study finding by Moges et al $(87.2 \%$ to $96.6 \%$, in 2019) [26], The geographical location, study time, and quantity of samples analyzed could all be factors in the reported discrepancy. Extended spectrum cephalosporin resistance was found in $17.5 \%$ of clinical Enterobacterales isolates studied in North America and Europe between 2016 and 2018 [40], the observed discrepancy could be attributed to the study's large geographical scope, infection control techniques used in those settings, the number of samples tested, and so on. 


\section{Conclusion and Recommendations}

The finding from this study revealed a high prevalence of Multidrug-resistant (MDR), Extremely drug-resistant (XDR), carbapenemase-producing gram-negative bacteria, particularly among Intensive care unit (ICU) patients at the health facility level This necessitates a robust laboratorybased antimicrobial resistance monitoring and infection prevention and control program.

\section{Limitations of the study}

The responsible genes for carbapenemase production were not genotypically assessed. Possible patient clinical impact was not assessed. Tigecycline, colistin, and Fosfomycin were not available and not used for AST.

\section{Acknowledgments}

My deepest appreciation would go to Ethiopian Public Health institute for their support of reagents and supplies to conduct this research, and Dr. Adane Bitew and Dr. Eyasu Tigabu for their commitment in reviewing this manuscript. 


\section{References}

1. Ventola CL. The Antimicrobial resistance crisis: part 1: causes and threats. Pharmacy and therapeutics. 2015 Apr;40(4):277.

2. Aminov RI. A brief history of the antimicrobial era: lessons learned and challenges for the future. Frontiers in microbiology. 2010 Dec 8; 1:134.

3. Rolain JM, Canton R, Cornaglia G. Emergence of Antimicrobial resistance: the need for a new paradigm. Clinical Microbiology and Infection. 2012 Jul 1;18(7):615-6.

4. Watkins RR, Bonomo RA. Overview: global and local impact of antimicrobial resistance. Infectious Disease Clinics. 2016 Jun 1;30(2):313-22.

5. Souza GL, Rocha RF, Carvalho HD, Oliveira CD, Leite EM, Silva EU, Giarola LG, Couto BR, Starling CE. 2475. Incidence of Multidrug-Resistant, Extensively Drug-Resistant and Pandrug-Resistant Gram-Negative Bacteria in Brazilian Intensive Care Units. InOpen Forum Infectious Diseases 2019 Oct 2 (Vol. 6).

6. Kieffer N, Nordmann P, Aires-de-Sousa M, Poirel L. High prevalence of carbapenemaseproducing Enterobacteriaceae among hospitalized children in Luanda, Angola. Antimicrobial agents and chemotherapy. 2016 Oct 1;60(10):6189-92.

7. Zhou L, Feng S, Sun G, Tang B, Zhu X, Song K, Zhang X, Lu H, Liu H, Sun Z, Zheng C. Extensively drug-resistant Gram-negative bacterial bloodstream infection in hematological disease. Infection and drug resistance. 2019; 12:481.

8. Magiorakos AP, Srinivasan A, Carey RT, Carmeli Y, Falagas MT, Giske CT, Harbarth S, Hindler JT, Kahlmeter G, Olsson-Liljequist B, Paterson DT. Multidrug-resistant, extensively drug-resistant and pan drug-resistant bacteria: an international expert proposal 
for interim standard definitions for acquired resistance. Clinical microbiology and infection. 2012 Mar 1;18(3):268-81.

9. Walther-Rasmussen J, Høiby N. OXA-type carbapenemases. Journal of Antimicrobial Chemotherapy. 2006 Mar 1;57(3):373-83.

10. Papp-Wallace KM, Bethel CR, Distler AM, Kasuboski C, Taracila M, Bonomo RA. Inhibitor resistance in the KPC-2 $\beta$-lactamase, a preeminent property of this class A $\beta$ lactamase. Antimicrobial agents and chemotherapy. 2010 Feb 1;54(2):890-7.

11. Raman G, Avendano EE, Chan J, Merchant S, Puzniak L. Risk factors for hospitalized patients with resistant or multidrug-resistant Pseudomonas aeruginosa infections: a systematic review and meta-analysis. Antimicrobial Resistance \& Infection Control. 2018 $\operatorname{Dec} ; 7(1): 1-4$.

12. CLSI. Performance Standards for Antimicrobial Susceptibility Testing. 30th ed. CLSI supplement M100. Wayne, PA: Clinical and Laboratory Standards Institute; 2020.

13. CLSI. Performance Standards for Antimicrobial Susceptibility Testing. 22th ed. CLSI supplement M100. Wayne, PA: Clinical and Laboratory Standards Institute; 2012.

14. van der Zwaluw K, de Haan A, Pluister GN, Bootsma HJ, de Neeling AJ, Schouls LM. The carbapenem inactivation method (CIM), a simple and low-cost alternative for the Carba NP test to assess phenotypic carbapenemase activity in gram-negative rods. PloS one. $2015 \mathrm{Mar}$ $23 ; 10(3): \mathrm{e} 0123690$.

15. Pierce VM, Simner PJ, Lonsway DR, Roe-Carpenter DE, Johnson JK, Brasso WB, Bobenchik AM, Lockett ZC, Charnot-Katsikas A, Ferraro MJ, Thomson RB. Modified carbapenem inactivation method for phenotypic detection of carbapenemase production among Enterobacteriaceae. Journal of clinical microbiology. 2017 Aug 1;55(8):2321-33. 
16. CLSI. Performance Standards for Antimicrobial Susceptibility Testing. 27th ed. CLSI supplement M100. Wayne, PA: Clinical and Laboratory Standards Institute; 2017.

17. Jing X, Zhou H, Min X, Zhang X, Yang Q, Du S, Li Y, Yu F, Jia M, Zhan Y, Zeng Y. The simplified carbapenem inactivation method (sCIM) for simple and accurate detection of carbapenemase-producing Gram-negative bacilli. Frontiers in microbiology. 2018 Oct 30; 9:2391.

18. Use I, Of C, Packages THE, Samples K, Procedure T, Results ETHE. MIC Test Strip Technical Sheet KPC. 2015;7-8.

19. Beyene D, Bitew A, Fantew S, Mihret A, Evans M. Multidrug-resistant profile and prevalence of extended-spectrum $\beta$-lactamase and carbapenemase production in fermentative Gram-negative bacilli recovered from patients and specimens referred to National Reference Laboratory, Addis Ababa, Ethiopia. PloS one. 2019 Sep 25;14(9):e0222911.

20. Gashaw M, Berhane M, Bekele S, Kibru G, Teshager L, Yilma Y, Ahmed Y, Fentahun N, Assefa H, Wieser A, Gudina EK. Emergence of high drug resistant bacterial isolates from patients with health care associated infections at Jimma University medical center: a cross sectional study. Antimicrobial Resistance \& Infection Control. 2018 Dec;7(1):1-8.

21. Bitew A, Tsige E. High Prevalence of Multidrug-Resistant and Extended-Spectrum $\beta$ Lactamase-Producing Enterobacteriaceae: A Cross-Sectional Study at Arsho Advanced Medical Laboratory, Addis Ababa, Ethiopia. Journal of tropical medicine. 2020 Apr $30 ; 2020$. 
22. Abbas S, Sabir AU, Khalid N, Sabir S, Khalid S, Haseeb S, Khan MN, Ajmal WM, Azhar F, Saeed MT. Frequency of Extensively Drug-Resistant Gram-Negative Pathogens in a Tertiary Care Hospital in Pakistan. Cureus. 2020 Dec;12(12).

23. Eshetie S, Unakal C, Gelaw A, Ayelign B, Endris M, Moges F. Multidrug resistant and carbapenemase producing Enterobacteriaceae among patients with urinary tract infection at referral Hospital, Northwest Ethiopia. Antimicrobial resistance and infection control. 2015 Dec;4(1):1-8.

24. Legese MH, Weldearegay GM, Asrat D. Extended-spectrum beta-lactamase-and carbapenemase-producing Enterobacteriaceae among Ethiopian children. Infection and drug resistance. $2017 ; 10: 27$.

25. Moges F, Gizachew M, Dagnew M, Amare A, Sharew B, Eshetie S, Abebe W, Million Y, Feleke T, Tiruneh M. Multidrug resistance and extended-spectrum beta-lactamase producing Gram-negative bacteria from three Referral Hospitals of Amhara region, Ethiopia. Annals of clinical microbiology and antimicrobials. 2021 Dec;20(1):1-2.

26. Moges F, Eshetie S, Abebe W, Mekonnen F, Dagnew M, Endale A, Amare A, Feleke T, Gizachew M, Tiruneh M. High prevalence of extended-spectrum beta-lactamase-producing gram-negative pathogens from patients attending Felege Hiwot Comprehensive Specialized Hospital, Bahir Dar, Amhara region. PloS one. 2019 Apr 15;14(4):e0215177.

27. Alemayehu T, Asnake S, Tadesse B, Azerefegn E, Mitiku E, Agegnehu A, Nigussie N. Phenotypic Detection of Carbapenem-Resistant Gram-Negative Bacilli from a Clinical Specimen in Sidama, Ethiopia: A Cross-Sectional Study. Infection and Drug Resistance. $2021 ; 14: 369$. 
28. Okoche D, Asiimwe BB, Katabazi FA, Kato L, Najjuka CF. Prevalence and characterization of carbapenem-resistant Enterobacteriaceae isolated from Mulago National Referral Hospital, Uganda. PLoS One. 2015 Aug 18;10(8):e0135745.

29. López-Hernández I, Delgado-Valverde M, Fernández-Cuenca F, López-Cerero L, Machuca J, Pascual Á. Carbapenemase-Producing Gram-Negative Bacteria in Andalusia, Spain, 2014-2018. Emerging infectious diseases. 2020 Sep;26(9):2218.

30. Alebel M, Mekonnen F, Mulu W. Extended-Spectrum $\beta$-Lactamase and Carbapenemase Producing Gram-Negative Bacilli Infections Among Patients in Intensive Care Units of Felegehiwot Referral Hospital: A Prospective Cross-Sectional Study. Infection and Drug Resistance. 2021; 14:391.

31. Teklu DS, Negeri AA, Legese MH, Bedada TL, Woldemariam HK, Tullu KD. Extendedspectrum beta-lactamase production and multi-drug resistance among Enterobacteriaceae isolated in Addis Ababa, Ethiopia. Antimicrobial Resistance \& Infection Control. 2019 $\operatorname{Dec} ; 8(1): 1-2$.

32. Hemmati H, Hasannejad-Bibalan M, Khoshdoz S, Khoshdoz P, Kalurazi TY, EbrahimSaraie HS, Nalban S. Two years study of prevalence and antibiotic resistance pattern of Gram-negative bacteria isolated from surgical site infections in the North of Iran. BMC research notes. 2020 Dec;13(1):1-6.

33. Biset S, Moges F, Endalamaw D, Eshetie S. Multi-drug resistant and extended-spectrum $\beta$ lactamases producing bacterial uropathogens among pregnant women in Northwest Ethiopia. Annals of Clinical Microbiology and Antimicrobials. 2020 Dec; 19:1-9. 
34. Gashe F, Mulisa E, Mekonnen M, Zeleke G. Antimicrobial Resistance Profile of Different Clinical Isolates against Third-Generation Cephalosporins. Journal of pharmaceutics. 2018 Sep 9; 2018:5070742-.

35. Breurec S, Bouchiat C, Sire JM, Moquet O, Bercion R, Cisse MF, Glaser P, Ndiaye O, Ka S, Salord H, Seck A. High third-generation cephalosporin resistant Enterobacteriaceae prevalence rate among neonatal infections in Dakar, Senegal. BMC infectious diseases. 2016 Dec;16(1):1-7.

36. Patolia S, Abate G, Patel N, Patolia S, Frey S. Risk factors and outcomes for multidrugresistant Gram-negative bacilli bacteremia. Therapeutic advances in infectious disease. 2018 Jan;5(1):11-8.

37. Nseir S, Di Pompeo C, Cavestri B, Jozefowicz E, Nyunga M, Soubrier S, Roussel-Delvallez M, Saulnier F, Mathieu D, Durocher A. Multiple-drug-resistant bacteria in patients with severe acute exacerbation of chronic obstructive pulmonary disease: Prevalence, risk factors, and outcome. Critical care medicine. 2006 Dec 1;34(12):2959-66.

38. Huang SF, Chang JS, Sheu CC, Liu YT, Lin YC. An antibiotic decision-making tool for patients with pneumonia admitted to a medical intensive care unit. International journal of antimicrobial agents. 2016 Sep 1;48(3):286-91.

39. Chen G, Xu K, Sun F, Sun Y, Kong Z, Fang B. Risk Factors of Multidrug-Resistant Bacteria in Lower Respiratory Tract Infections: A Systematic Review and Meta-Analysis. Canadian Journal of Infectious Diseases and Medical Microbiology. 2020 Jun 30;2020. 
40. Belley A, Morrissey I, Hawser S, Kothari N, Knechtle P. Third-generation cephalosporin resistance in clinical isolates of Enterobacterales collected between 2016-2018 from USA and Europe: genotypic analysis of $\beta$-lactamases and comparative in vitro activity of cefepime/enmetazobactam. Journal of global antimicrobial resistance. 2021 Jun 1;25:93101. 


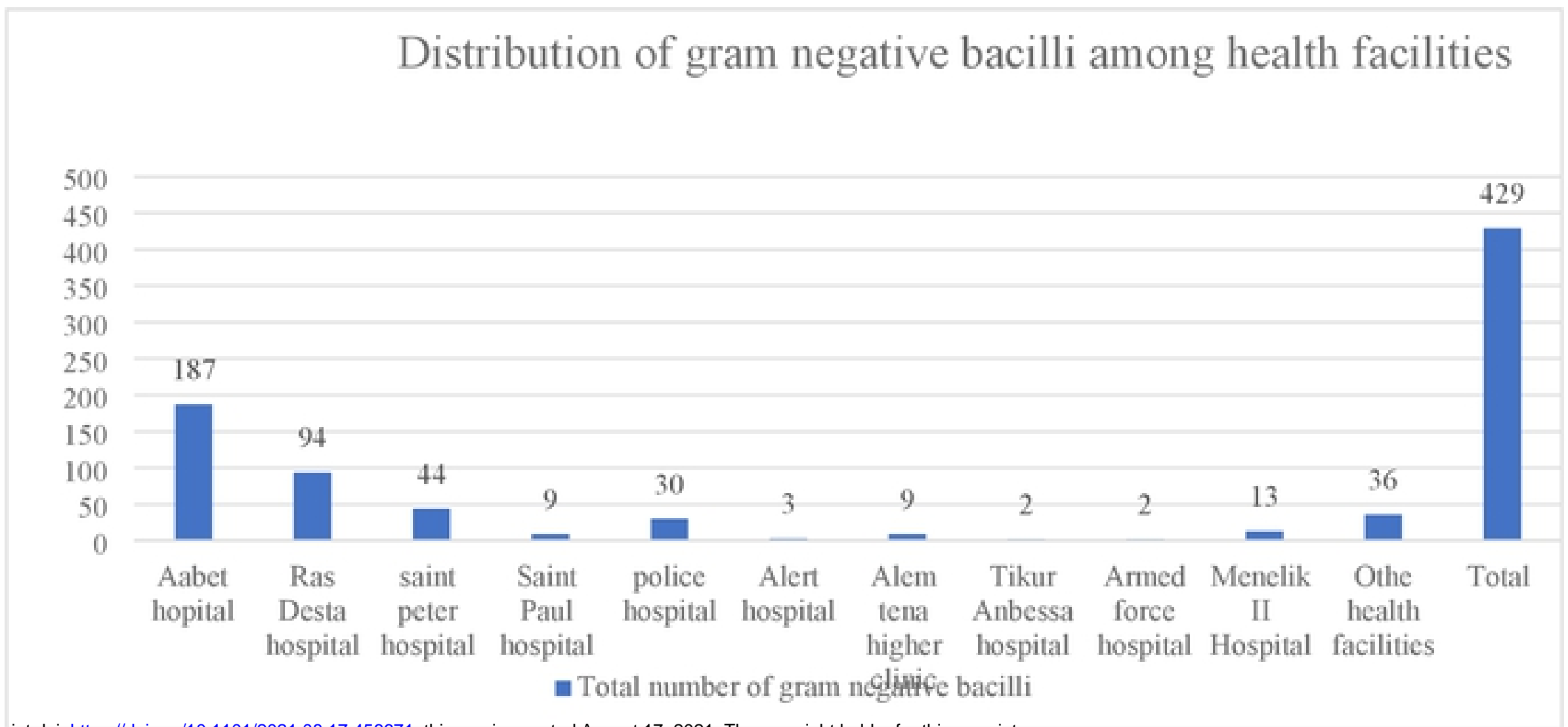

bioRxiv preprint doi: https://doi. org/10.1101/2021.08.17.456671; this version posted August 17, 2021. The copyright holder for this preprint (which was not certified by peer review) is the author/funder, who has granted bioRxiv a license to display the preprint in perpetuity. It is made

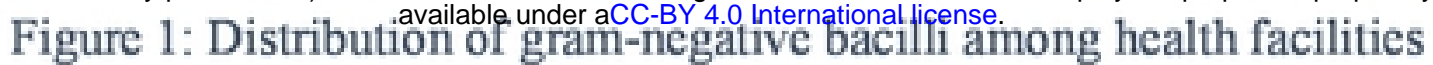

Figure 


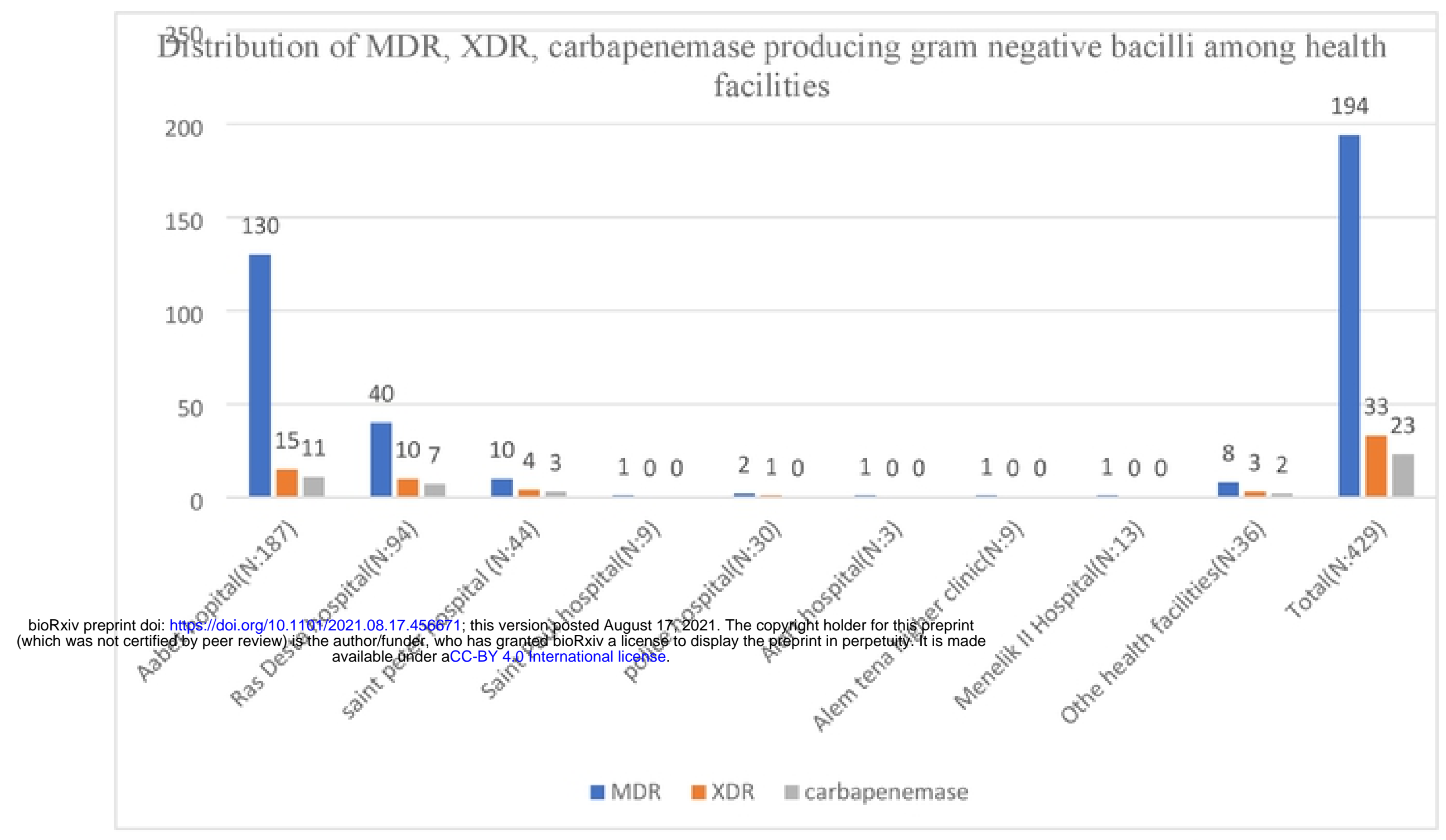

Figure 2: Distribution of MDR, XDR, carbapenemase-producing gram-negative bacilli among health facilities 